\title{
Study on Optimization of Power Communication Network Topology
}

\section{Based on Complex Network Theory}

\author{
QU Bei-bei ${ }^{1, a}$ \\ ${ }^{1}$ State Grid Information \& Telecommunication Company of SEPC, Taiyuan, Shanxi 030001,China \\ ${ }^{\mathrm{a}}$ email
}

Keywords: Complex Network; Power Communication Network; Topology Optimization

\begin{abstract}
With the increasingly complex structure of power communication network and diversified business needs, the demand of performance optimization in power communication network is more and more urgent. Based on complex network theory, this paper proposes a topology optimization algorithm of node betweenness plus matrix, which is proved to be effective in enhancing the ability of the network to resist selective attacks, improving the bandwidth utilization efficiency of key nodes and reducing the probability of link congestion.
\end{abstract}

\section{Introduction}

In recent years, the application of smart grid is gradually being popularized, and the characteristics of power grid informatization, automation and interaction are becoming more and more prominent, and the power communication network, which plays a strong supporting role in the grid, is undergoing profound changes. With the deep penetration of information communication technology with power production and management, the power grid is increasingly dependent on the power communication network, and the reliability and transmission performance of communication networks have become the key factors influencing the safe and stable operation of power network.

With the increasing complexity of network topology and more diversified business needs, load imbalance problem in power communication network is becoming more and more serious. Since the special properties of the power communication network topology and traffic flow have not been taken into account, most of the traditional optimization strategy is difficult to be directly used. Based on complex network theory, this paper proposes a topology optimization algorithm of node betweenness plus matrix. Comparative analysis results in simulation test shows that this algorithm can effectively enhance the ability of the network to resist selective attack, and also delay the effectiveness dump phenomenon. 


\section{Optimization Algorithm for Power Communication Network Topology}

Topology Optimization Process. Robust and fragile are important features of small-world networks. Utilize complex network theory to carry out robustness and vulnerability analysis on power communication network, to examine the network' fault tolerance and survivability when subjected to random attacks, and thus optimize and adjust the network topology according to the optimization strategy and objectives.

Optimization algorithmic process for power communication network topology based on complex network theory is shown as Fig. 1.

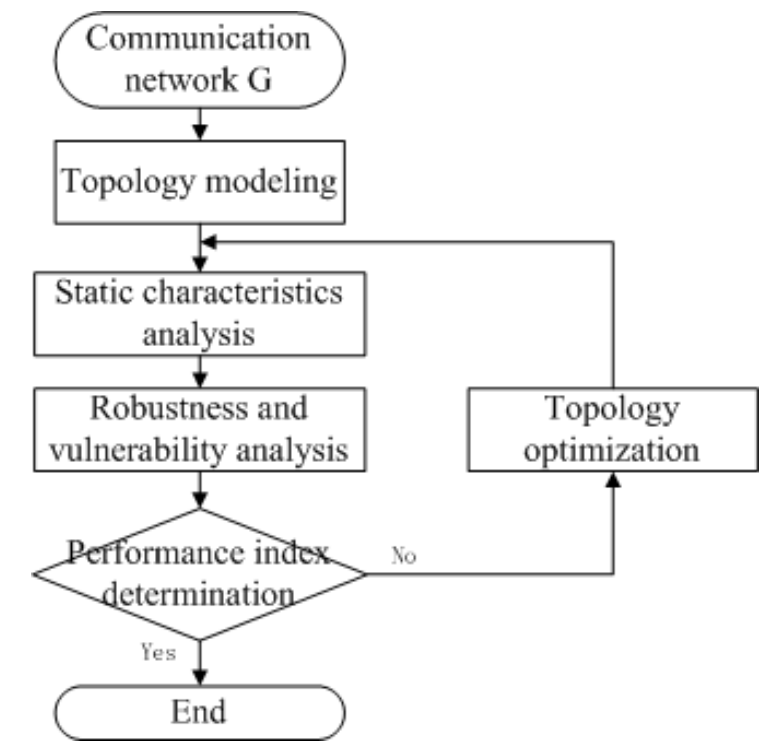

Fig.1. Topology optimization process

The objective of the network topology optimization algorithm is to increase the number of edges in the network while maintain the number of nodes. We introduce betweenness to represents the number of shortest paths passing through some certain node $v_{i}$ or edge $e_{i}$, and its value depicts the importance of the node $v_{i}$ and edge $e_{i}$ in network transmission: the node with larger betweenness generally indicates that it takes on more forwarding business in the actual network communication, so it needs to be based on a higher forwarding performance (such as port high speed, or multi-port device); the edge with larger betweenness also takes on more forwarding business and requires greater bandwidth to achieve high speed transmission and avoid congestion. It is thus clear that betweenness is an important parameter to reflect the traffic load of network equipment.

Topology Optimization Algorithm Based on Node Betweenness Plus Matrix. Power communication network is to establish a network connection based on business needs, so its nodes

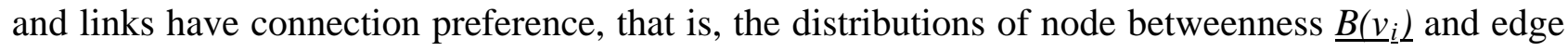
betweenness $B\left(e_{i}\right)$ are not uniform. By selecting the appropriate node to increase the link, we can effectively shorten the network diameter and reduce the forwarding speed. Based on the concept of node betweenness, this paper propose one network topology optimization algorithm based on node betweenness plus matrix, to realize the optimization and promotion of existing network performance, and effectively suppress the sudden drop in the performance of the network under random attack and selective attack.

The topology optimization algorithm flow is shown in Fig. 2. 


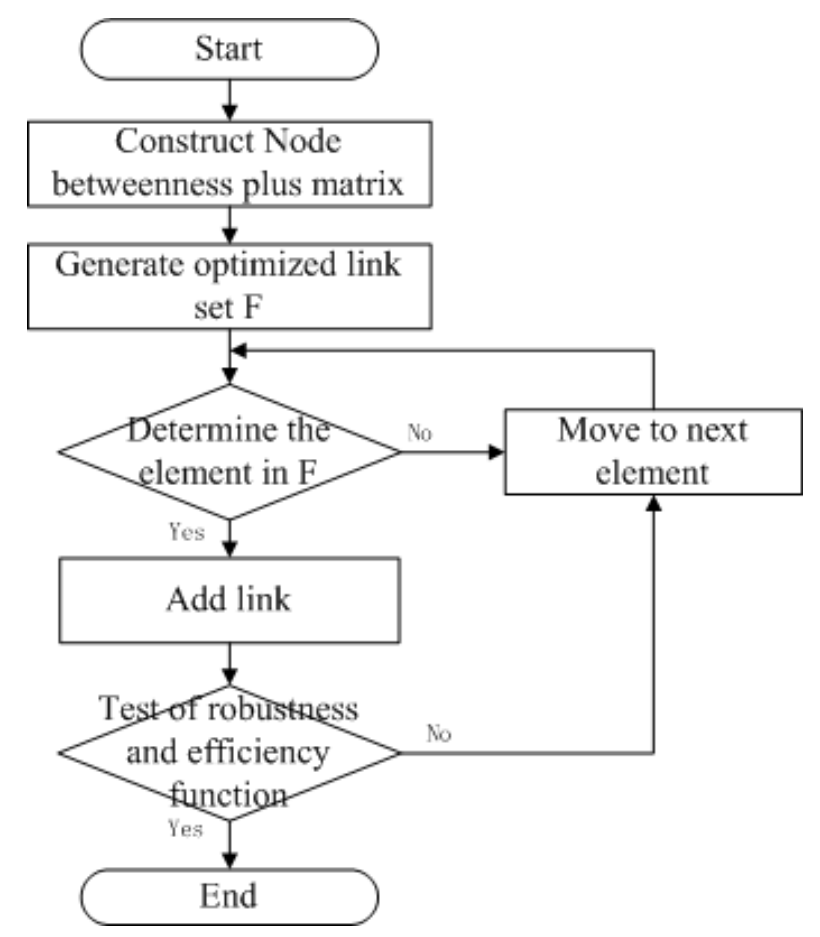

Fig.2. Topology optimization algorithm flow

The specific steps are as follow:

(1) Calculate the betweenness $b\left(v_{i}\right)$ of each node in the network, and then obtain node betweenness vector of the network: $\bar{B}=\left[b\left(v_{1}\right), b\left(v_{2}\right), \ldots, b\left(v_{n}\right)\right]^{T}$.

(2) Construct the node betweenness plus matrix $\left[\bar{B}^{+}\right]_{m}, m=C_{n}^{2}$, the matrix element ${\overline{B_{k}}}^{+}=b\left(v_{i}\right)+\left(v_{j}\right)$, and $k=(i-1) * n+j$.

(3) According to bubble sorting, sort $\left[\bar{B}^{+}\right]_{m}$ in descending order, and generate the descending node betweenness plus matrix $\left[\bar{B}^{+}\right]_{m}^{\prime}$; the node pair arranged before $\left[\bar{B}^{+}\right]^{\prime} m$ is of larger betweenness bonus.

(4) Based on the descending node betweenness plus matrix $\left[\bar{B}^{+}\right]_{m}^{\prime}$, generate optimized link set $F=\left[f_{1}, f_{2}, \ldots . ., f_{m}\right]$, and $f_{k}=f\left(v_{i}, v_{j}\right)$, thus generate the interconnected link between the corresponding node of element $\mathrm{b}(\mathrm{k})$ of $\left[\bar{B}^{+}\right]^{\prime} m$ and $\left(v_{i}, v_{j}\right)$.

(5) Construct the optimized node set $G=\left[g_{1}, g_{2}, \ldots \ldots, g_{n}\right]$, all the elements are initialized as 0 ; set the variable $\mathrm{p}=1, \mathrm{q}=1$.

(6) In order to avoid excessive concentration of the added links in the optimization process, we introduce duplicate nodes avoidance mechanism, of which specific realization way is: determine 
whether it meets $f p=f\left(v_{i}, v_{j}\right) \notin E$, and $v_{i} \notin G, v_{j} \notin G$, if so, then increase the link $\mathrm{e}\left(v_{i}, v_{j}\right)$, and meanwhile $g_{q}=v_{i}, g_{q+1}=v_{j}, q=q+2$; if not, move to the next element, and repeat the step (6).

(7) Calculate connectivity robustness $R$ and efficiency function $E$ of the network. If it achieves the goal of optimization, then terminate the network optimization algorithm; otherwise, $p=p+1$, jump to step (6) and add next required link for ongoing optimization, until the network performance meets the requirements.

Based on the optimization algorithm we propose, only by adding limited links, it is not hard to achieve the network optimization objective. However in actual application, we should also comprehensively consider the network status, cable physical topology and the actual position of the nodes, to determine the final rational optimization scheme.

\section{Optimization effect analysis}

According to the network topology optimization process, taking the power communication network topology in a certain area as example, we carry out the robustness and vulnerability analysis, and based on the topology optimization algorithm of node betweenness plus matrix to optimize it, and then test the optimization effect of this algorithm by comparative analysis.

Researchers have made a lot of research on the topology optimization problem of complex networks, and proposed a variety of optimization algorithms. For example, Wu Jun ever proposed a topology optimization algorithm based on the characteristic value of the network adjacency matrix, and proved to have obvious effect in enhancing the invulnerability of complex networks. In this paper, we adopt this algorithm as a contrast, to verify the effect of our algorithm.

In the test experiment, we add 8 links in the network g for topology optimization, and observe the changes in network performance after optimization, comparing with the selected algorithm. The simulation result of these two optimization algorithm is shown in Fig. 3:

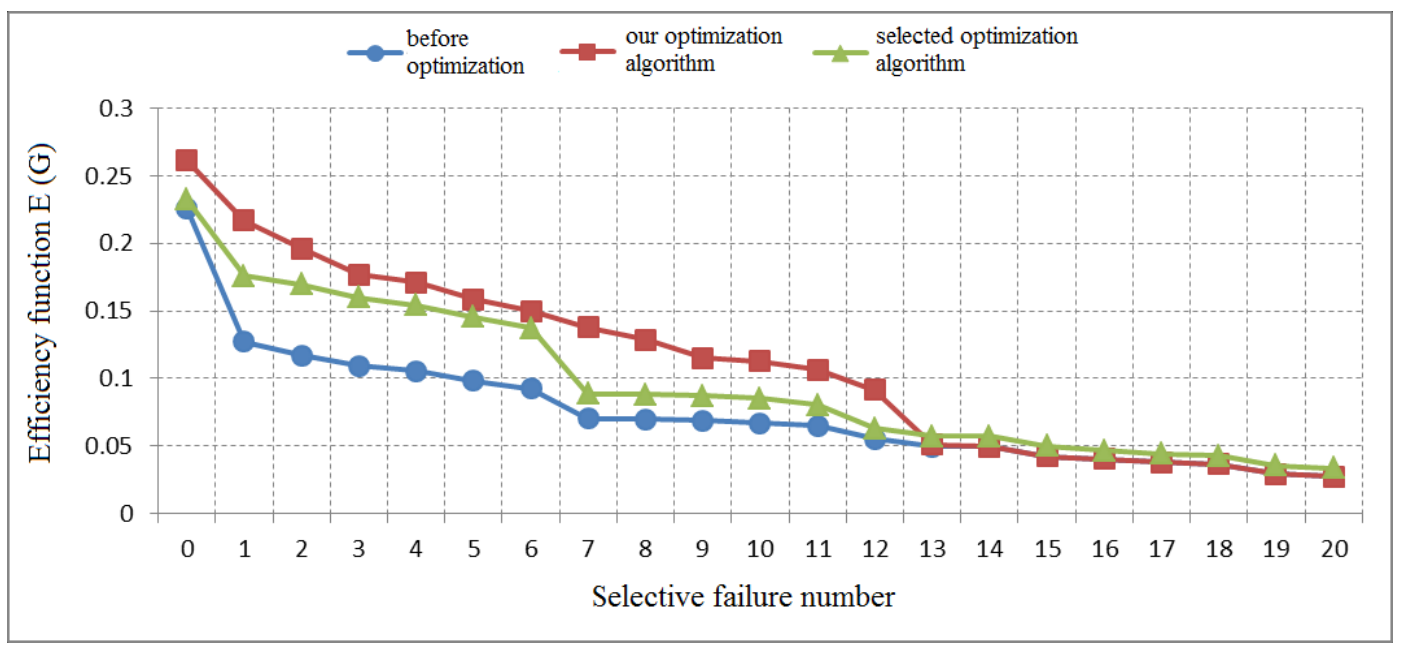




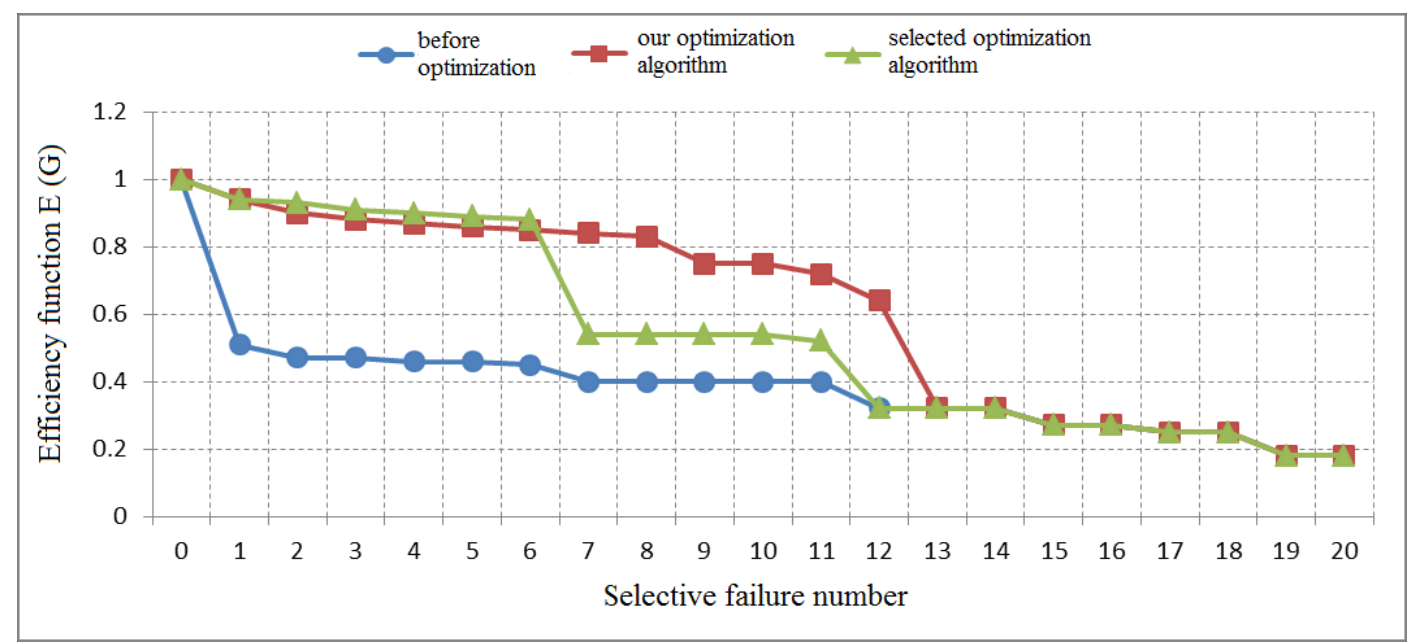

Fig.3. Topology optimization algorithm flow

From the figure we can learn that, in terms of node betweenness selective fault, our algorithm is superior to the selected algorithm. The efficiency function and connectivity robustness of the network is greatly improved compared to the original network. Moreover, the optimization algorithm based on node betweenness plus matrix can not only enhance the ability of the network to resist selective attacks, but also effectively delay the effectiveness dump phenomenon.

\section{References}

[1] Shi J H, Zong R, Liu Y M, et al. Study on the invulnerability and topology optimization of power communication network[J]. Telecommunications for Electric Power System, 2009.

[2] Zhou J, Zi-Wei H U, Liu G J, et al. Research on Business Requirement Analysis and Topology Planning of Backbone Power Communication Network[J]. Study on Optical Communications, 2016.

[3] Xing N. Optimization Algorithm of Power Communication Network Based on the Equivalent Network[J]. Applied Mechanics \& Materials, 2014, 596(12):653-658.

[4] Guo J, Wang D R. Vulnerability analysis on power communication network based on complex network theory[J]. Telecommunications for Electric Power System, 2009.

[5] Hu J, Yu J, Cao J, et al. Topological interactive analysis of power system and its communication module: A complex network approach[J]. Physica A Statistical Mechanics \& Its Applications, 2014, 416:99-111. 